\title{
Optimal Frequency Control Management of Grid Integration PV/Wind/FC/Storage Battery Based Smart Grid Using Multi Objective Particle Swarm Optimization MOPSO and Model Predictive Control (MPC)
}

\author{
Adel Elgammal and Tagore Ramlal
}

\begin{abstract}
This article forecasts the performance of smartgrid electrical transmission systems and integrated battery/FC/Wind/PV storage system renewable power sources in the context of unpredictable solar and wind power supplies. The research provided a hybrid renewable energy sources smart grid power system electrical frequency control solution using adaptive control techniques and model predictive control (MPC) based on the Multi-Objective Practical Swarm Optimization Algorithm MOPSO. To solve the problems of parameter tuning in Load Frequency Control, the suggested adaptive control approach is utilized to accomplish on-line adjustment of the Load Frequency Control parameters. During the electrical grid's integration, the system under investigation is a hybrid Wind/PV/FC/Battery smart grid with variable demand load. To achieve optimal outcomes, all of the controller settings for various units in power grids are determined by means of a customized objective function and a particle swarm optimization method rather than a regular objective function with fluctuating restrictions. To suppress the consumption and generation balance, MPCs were designed for each of the Storage Battery, Wind Turbine Generation, and the model Photovoltaic Generation. In addition, demand response (real-time pricing) was used in this scheme to reduce the load frequency by adjusting the controlled loads. The suggested control strategy is evaluated in the Simulink /MATLAB environment in order to analyse the suggested approach's working in the power system, as well as its effectiveness, reliability, robustness, and stability. The simulation findings show that the proposed control method generally converges to an optimal operating point that minimises total user disutility, restores normal frequency and planned tie-line power flows, and maintains transmission line thermal restrictions. The simulation results further indicate that the convergence holds even when the control algorithm uses inaccurate system parameters. Finally, numerical simulations are used to illustrate the proposed algorithm's robustness, optimality, and effectiveness. In compared to previous methodologies, the system frequency recovers effectively and efficiently in the event of a power demand disturbance, as demonstrated. A sensitivity test is also performed to assess the suggested technique's effectiveness.
\end{abstract}

Index Terms - Fuel Cells, Photovoltaic, Wind Turbines, Smart grid, Load Frequency Control, AC/DC conversion, Multi-Objective Practical Swarm Optimization Algorithm, Model predictive control; Renewable energy sources; Battery Storage System.

Submitted on June 21, 2021

Published on July 12, 2021.

Adel Elgammal, The University of Trinidad and Tobago UTT, Utilities Engineering, Point Lisas Campus, Trinidad and Tobago.

(e-mail: adel_elgammal2000@yahoo.com)

\section{INTRODUCTION}

Power systems are facing new issues in balancing supply and demand and sustaining normal frequency as the use of renewable energy supplies grows [1]. The general power system is a complicated electrical linkage which includes generating, transmission, and distribution networks, as well as loads that are scattered across the network across a vast geographic region [2]. In the case of a linked power system, a modest rapid load shift in any of the regions causes the frequencies of each region to fluctuate, along with the power in the tie line [3]. The complexity of the power system grows in tandem with the rise of industry. The active power of a system determines its frequency, whereas the reactive power determines its voltage. As a result, power system control can be divided into 2 distinct challenges. One focuses on active power and frequency management, known as load frequency control, while the former deals with reactive power and voltage regulation, known as voltage regulation [4]. Welldesigned controllers are necessary to maintain the power system's stability and assure its dependable operation during system fluctuations [5]. In general, power imbalance between generation and demand leads electricity to be exchanged between different places, resulting in frequency variation in the power structure. In this case, LFC is a primary response to the imbalance, with the goal of restoring the interchanging power and frequency to their planned levels [6]. The development of the power system's scale and complexity, on the one hand, and the growth of power consumption, on the other, demands innovative smart systems with various energy sources. Scientists are actively working on novel LFC ways to retain the interchanging power and frequency in predetermined quantities in the face of a variety of disruptions [7]. LFC of an interconnected power system involves connecting more than one area of control via tie lines. Tie line power deviation and frequency shift will occur if a sudden power fluctuation occurs in any control zone of a power system network. Large frequency variations can occasionally result in a "system blackout" [8]. Load Frequency Control is a system for balancing power generation and demand. The main purpose of the Load Frequency Control is to retain interarea tie-line power flows at pre-determined contract levels while maintaining system frequency at nominal levels.

Tagore Ramlal, The University of Trinidad and Tobago UTT, Utilities Engineering, Point Lisas Campus, Trinidad and Tobago. 
Alternatively, to maintain the connected power system's targeted power output (MW) and real frequency by controlling the change in tie line power between control zones [9]. The main goals of load frequency control are to maintain acceptable overshoot and settling time on t, to control the change in tie line power among control regions, to maintain power interchanges and frequency with adjacent control regions at specified values, to ensure frequency deviations zero steady-state error, and to regulate track load demands and frequency [10]. So, a load frequency control scheme is essentially an interconnected power system ' control system that has the ability of tie line powers back to original set point values or very close to set point values following a load shift and effectively bringing the frequencies of each region. This is accomplished through the use of traditional controls. However, traditional controllers have significant drawbacks, such as making it tough to find out the improvement of the integrator setting in response to variations in the operating point, ignoring the inherent nonlinearities of different power system components and being sluggish to operate [11]. In the power system LFC application, simple and traditional tuned controllers are currently frequently employed. Existing load frequency control system settings are often set based on trial and errors approaches, traditional methodologies and past experiences, and thus are unable to provide acceptable dynamic performance across a wide variety of load situations and operating circumstances. To accomplish a new trade-off between resilience and efficiency, control methods and novel modelling are thus necessary [12].

The advantages of an advanced control system over a traditional integrated controller are numerous. They are quicker than integrated controllers, and they also provide a greater stability response. Optimization control techniques and advanced control techniques (optimal compensator, optimal controller) have been used for LFC of two area power structures in this suggested study effort. The best controllers and compensators can operate in the presence of process noise and measurement and in the absence of complete state feedback. The IMC-PID controller has an enhanced reaction time and may be used in a variety of nonlinear situations [13]. In the process sector, Model Predictive Control (MPC) has been universally regarded as a valid control approach. It's a model-based control method that uses an optimization strategy to find the best control action at each sampling interval over a prediction horizon. The optimization criterion, also known as the objective function, is chosen to reduce control effort, punish system output departure from planned route, and to fulfil the controlled system constraints and dynamics. Furthermore, the MPC is an excellent option for power system control because it may include economic objectives into the optimization criterion [14], [15]. Due to the traditional demands of system security and reliability, the existence of economic and technological constraints, and the high degree of interconnection, operating the power system in the new environment will definitely be more difficult than in the past. In today's power system LFC work, simple and traditional tuned controllers are extensively employed. Existing load frequency control system settings are often set based on trial and errors approaches, traditional methodologies, and past experiences, and thus are unable to provide high dynamical performance across a wide range of operating circumstances and load scenarios. Control methodologies and novel modelling are thus essential in order to achieve a new trade-off between resilience and efficiency [16], [17].

With the goal of enhancing LFC performance, researchers have recently looked at modern control theory [18], neural networks [19], fuzzy system theory [20], and the ANFIS approach [21]. However, many recent approaches, such as the ones discussed above, come with a level of complexity that restricts their use. As a result, traditional PI and PID controllers are favoured, as they provide benefits such as a simple construction and high performance. In certain articles, intelligent algorithms are also employed to optimize the settings of PI/PID controllers. When contrasted to traditional and GA-based controllers, the Bacterial Foraging Optimization Algorithm was determined in [22]. [23] Uses the differential evolution (DE) method to optimize the controller's settings, and the results are compared to those of the BFOA and genetic algorithms. [24] Considers generation rate constraints for thermal and hydro units to create a single region including gas, hydro, and thermal units. For optimization, an optimum output feedback controller based solely on output state variables is provided. The majority of published research in this subject ignores practical limits and uncertainties, and instead proposes unworkable frameworks with sophisticated control structures, which may pose challenges in real-time applications. Using a novel optimization methodology, this work proposes an adaptive control strategy that performs on-line adjustments to the MPC parameters to cope with both fluctuations and load demand variations caused by renewable energy sources.

\section{DESCRIPTION OF THE PROPOSED PV/WIND/FC/BATTERY MICROGRID SYSTEM}

The suggested system for research is a single-area power system that includes wind turbines, fuel cells, solar panels, and battery storage. Fig. 1 depicts the system's linearized model, which is employed for simulation and optimum frequency control research. As demonstrated in Fig. 2, as first-order dynamical frequency models, simple dynamical models for energy storage systems (ESSs) and renewable energy resources were used. Although low-order dynamic models appear to be adequate, they are unable to account for all dynamic characteristics of frequency behaviour due to climate trends and parametric uncertainties, such as sun irradiation changes and wind power variation. Frequency control components can be divided into 2 types in general. These are primary control (for a several second) and secondary control (for nearly several seconds to several minute). Mechanical speed and inertia response governor control of rotating generators provide primary frequency control. Because of its rapid onset, the inertia response is sometimes regarded a different category from the main reaction. Because converters are far quicker than governors, the difference in frequency response is considerably more noticeable. Automatic generation control (AGC) provides secondary frequency response, and generators change their real power to return frequency to its nominal value based on signals supplied by Automatic generation control. As a result, spinning machines need many minutes to complete this 
operation, but converter-based frequency control may react much quicker, typically on a sub-second time scale. For the Active power control loop, Active power generating by unit is controlling in this loop mainly. In the simplest way, when a change in unit's active power is needed, new active power reference is set. If there is no change in grid frequency, only input of controller is difference between electrical active power of generation unit $(\mathrm{P})$ and reference of active power (Pref) : P- Pref $=\Delta \mathrm{P}$ which a MPC controller can be used for to make it zero by sending signal for change in active power of unit. There are limits for maximum and minimum power that a unit can deliver. Signal can be limited. For the frequency control loop; difference between electrical active power of generation unit $(\mathrm{P})$ and reference of active power (Pref): $\mathrm{P}$ - Pref $=\Delta \mathrm{P}$ and nominal frequency of grid (Fref) and real frequency of grid (f): $\mathrm{f}$-fref $=\Delta \mathrm{f}$. If $\Delta \mathrm{f}<0$, there should be $\Delta \mathrm{P}>0$; if $\Delta \mathrm{F}>0$, there should be $\Delta \mathrm{P}<0$ and if $\Delta \mathrm{f}=0$, there should be $\Delta \mathrm{P}=0$, so it seems best idea to have $\Delta \mathrm{f}+\Delta \mathrm{P}$ as input of MPC controller an try to make it equal to zero all time. Sudden or rapid change in frequency should not change active power suddenly so a rate limiter block is added before droop. Maximum frequency deviation that unit is expected to stay connected to grid and contribute in primary frequency control should be considered too so there will be a limiter first of all. One more thing is about how frequently unit may change its output power. The participation factor for every unit is used to determine how much each unit contributes to nominal loads; the factors for FC, Wind, Battery Storage System, and PV units are KFC, KPV, KWind, and KBSS, respectively. The goal is to have the sum of these variables equal one. PV modules are usually connected to the grid through an inverter and a boost converter. To get the most power out of PV modules, one of the maximum power point methods, which does this by changing the duty cycle of the boost converter, must be used. In this study, a MOPSO-based algorithm is used to track the largest power point. This approach is quite effective, and it can even track the highest power point in partially dim conditions. After calculating each module's maximum power, a part of that power can be allocated to reserved storage. It is necessary to study elements that impact PV power in order to assign a part of the generated power by PV systems. Three factors impact PV module power: temperature, irradiation, and output current (or voltage). If the irradiance and temperature remain constant, changing the output voltage of PV modules can affect their power. The MOPSO algorithm initially specifies the maximum power (Pmppt) and voltage (Vmppt). The quantity of reserve power for each module is then determined, and modules must now run at PFra rather than Pmppt. In other words, the duty cycle of converters is altered such that the boost converter's output voltage is VFra rather than Vmppt. It's worth noting that the tiny signal dynamic model for inverters used in solar panel structures has been upgraded in order to mimic various components of this distributed generation effectively. In reality, the solar panels output power may be calculated using the MPPT curve and then transmitted to the utility grid using an interface device and an inverter-based topology.

A continuous droop is offered as a representation of virtual inertia of rotating masses in the suggested dynamic WTG model (RWT). This droop can play a pivotal role in improving frequency deviation and compelling generating units to inject active power while some critical events occur. Two separate devices are presented to acquire frequency deviation more precisely: a washout filter, which are a highpass filter and a measuring device. Energy storage systems are essential for providing enough energy for isolated MG in a short amount of time while ensuring system stability. It is often assumed that charging BESS takes a certain amount of time, hence temporal constants for various components utilised in the building of the energy storage system appear to be essential. BESSs have been viewed as first order models in many earlier researches, which has led to the disregard of some dynamic behaviour of BESSs. In order to explore more accurate dynamic behaviour of BESSs, the BESS transfer function can be described as a three-block schematic that includes a converter block, a command delay device, and a frequency measuring device. During frequency research, the fuel cell is widely recognised as a green energy source. Three unique dynamic blocks are introduced, including an inverter architecture that can be used to convert DC voltages to AC voltages. An interconnection device, which efficiently connects distributed generation to the power grid, is another important component. Because there is frequently a common MPC controller between the load control loop and the frequency control loop, these two loops are referred to as the load-frequency control loop.

\section{OBJECTIVE FUNCTIONS}

The primary purpose of LFC is to regulate the controllers' settings such that the interchanging power and frequency stay within predefined limits. As a result, an objective function must be created to achieve desirable parameters like reduced inaccuracy, lower overshoot and faster settling time. Many prior assessments have utilised the integral time multiplied absolute error as an objective function, which is given by:

$$
I T A E=\int_{0}^{t} t|e(t)| d t
$$

where, the system error is denoted by e(t). Although integral time multiplied absolute error can improve settling time, it is unable to reduce overshoot effectively. As a result, this paper proposes a modified target function that can reduce the system response's overshoot while simultaneously shortening its settling time. Where, $\Delta$ Ptie is interchanging power between areas, and $\Delta \mathrm{f}$ is frequency deviation. The performance indices include the steady-state error of $\Delta \mathrm{f}$ and $\Delta \mathrm{Ptie}$, settling time of $\Delta \mathrm{f}$ and $\Delta \mathrm{Ptie}$, the rising time of of $\Delta \mathrm{f}$ and $\Delta \mathrm{Ptie}$, the overshoot of $\Delta \mathrm{f}$ and $\Delta \mathrm{Ptie}$, the integral of time multiplied square error, the integral of square error, the integral of time multiplied absolute value of the error, and the integral of absolute value of the error. 


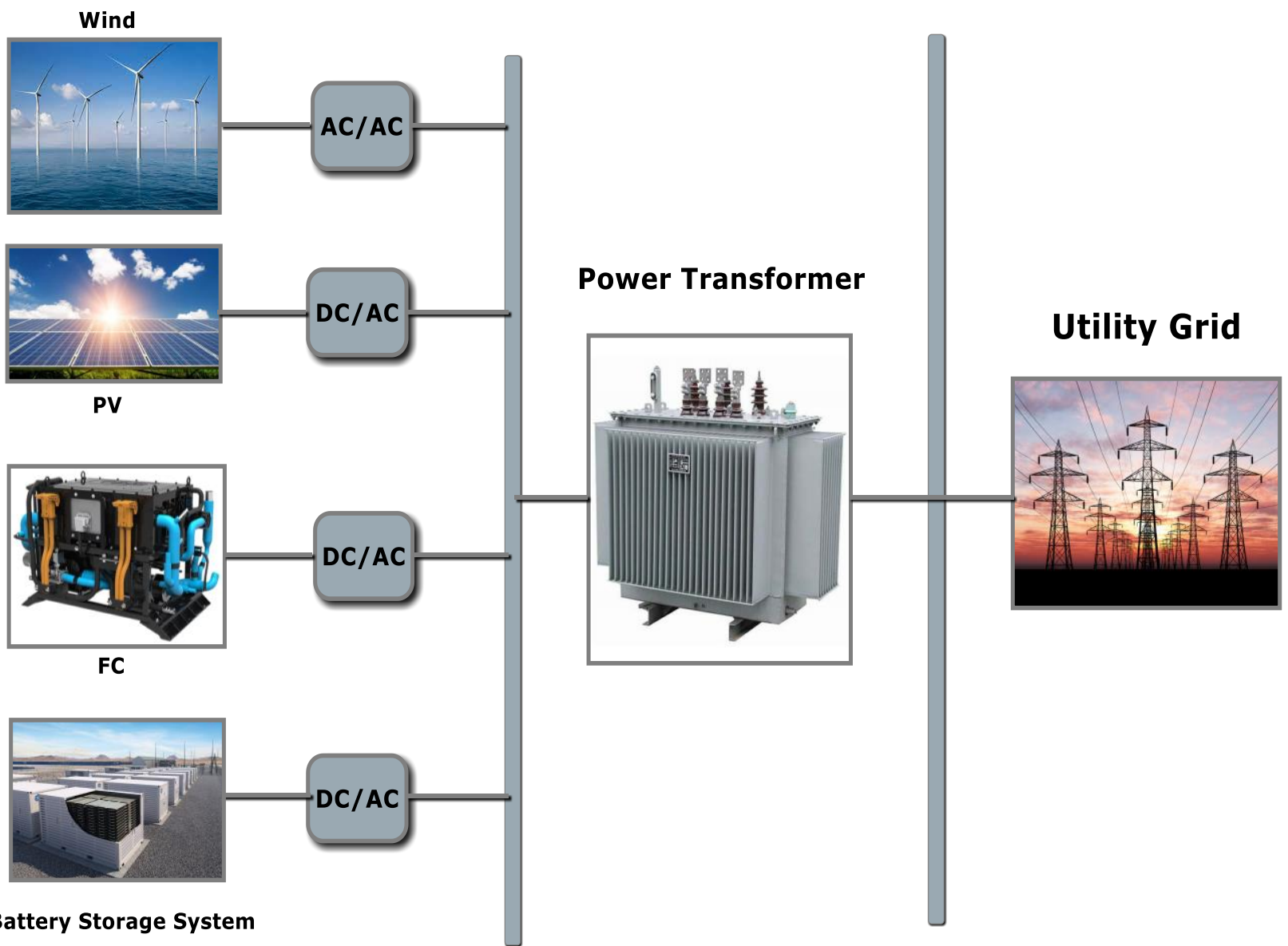

Fig. 1. The suggested microgrid understudy for load-frequency control comprising FC, Wind, PV units, and Battery Storage System.

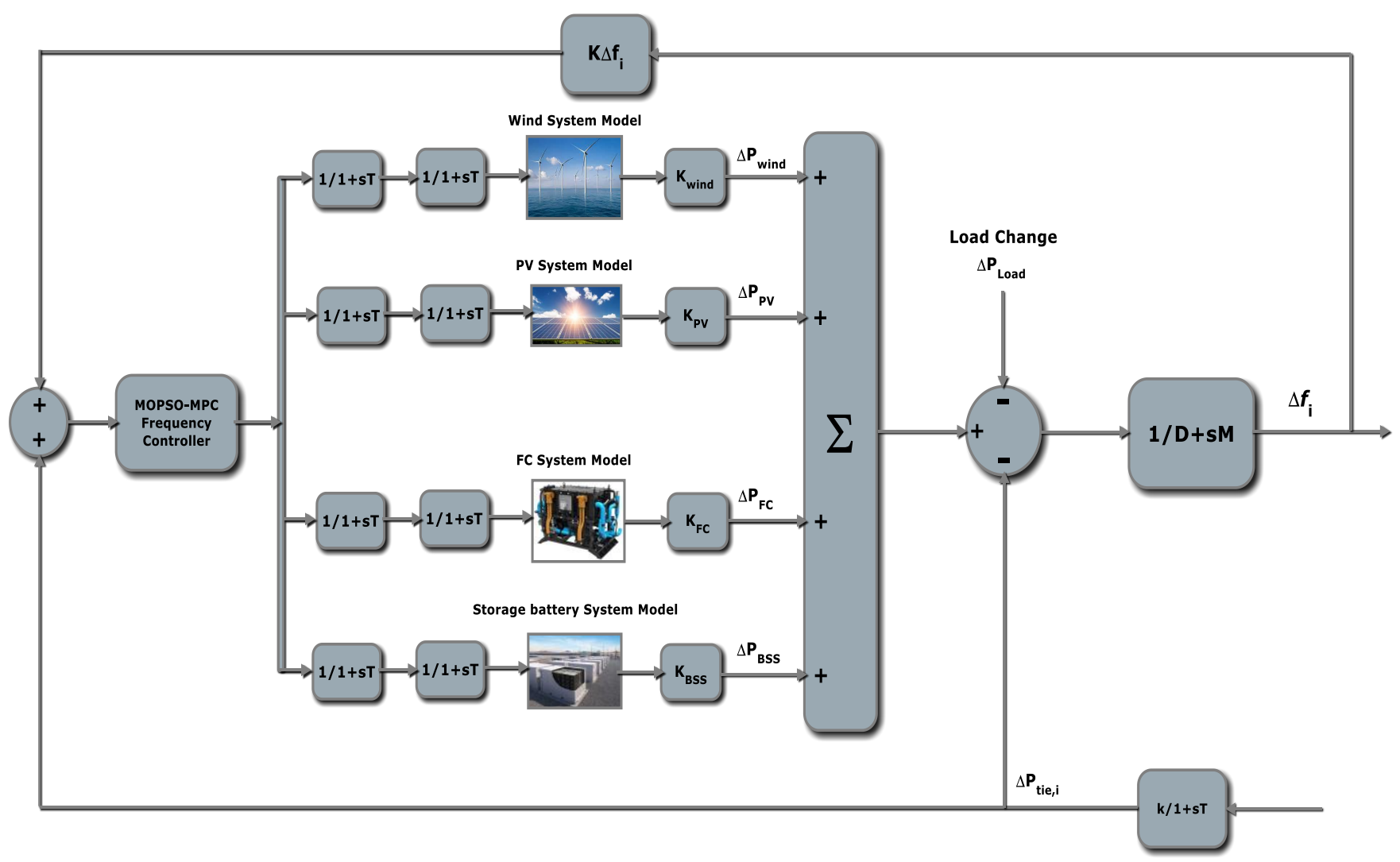

Fig. 2. Block diagram of the frequency domain of the microgrid under-study. 


$$
\begin{aligned}
& J_{1}=\int_{0}^{t}\left(\left|\Delta f_{i}\right|+\left|\Delta P_{\text {tie }}\right|\right) d t \\
& J_{2}=\int_{0}^{t} t\left(\left|\Delta f_{i}\right|+\left|\Delta P_{\text {tie }}\right|\right) d t \\
& J_{3}=\int_{0}^{t}\left((\Delta f)^{2}+\left(\Delta P_{\text {tie }}\right)^{2}\right) d t \\
& J_{4}=\int_{0}^{t} t\left((\Delta f)^{2}+\left(\Delta P_{\text {tie }}\right)^{2}\right) d t
\end{aligned}
$$

\section{Simulation Results}

To determine the efficacy and robustness of the work, the performance of controllers adjusted by objective functions produced based on MOPSO and MOGA is compared to examine the influence of various criteria used to construct objective functions on the best solutions to the LFC issue. Figure 3 depicts the simulation results for frequency deviation and active power deviation responses when a $2 \%$ step increase in demand is treated as a disturbance load increase with and without the use of the MOPSO optimization approach. As we discovered, the frequency fluctuation MOPSO control strategy has high performance, even if there are still some disturbances induced by solar radiation, but it demonstrates the efficacy and durability of a renewable power system frequency control strategy.

The MOGA and MOPSO effects result in a smaller overshoot and a shorter settling time, as demonstrated in this diagram. In this scenario, different controllers are used to compare frequency overshoot, and settling time of the frequency signal and the maximum frequency deviation. The maximum deviation of tie-lines active power and settling time are also provided for additional inquiry. According to the data, MOGA and MOPSO controllers reduce settling time and maximum overshoot more than MPC and PID controllers. According to the findings, the MOPSO and MOGA controllers offer the appropriate level of the MG outcome $(\Delta \mathrm{f})$ in terms of settling time and transient variations. The recommended methodology, on the other hand, can yield significantly lower $\Delta \mathrm{f}$ variations with considerably faster result specifications. In other words, the real-time outcomes display that the proposed strategy is more successful at dealing with the unpredictability of wind and PV than the model-based alternatives evaluated. The stochastic nature of renewable energy sources, for example solar and wind energy, causes MG frequency oscillations. Because of its responsibility to maintain frequency in its planned value in normal situations and in the event of a very modest load variation, LFC plays a significant role in the MG system under these situations. However, without a solid analytical model, controlling hybrid power system operations in unknown situations is a more difficult task. This necessitates the use of sophisticated control approaches to meet the system's stability needs. To test the stability of the suggested strategy, a sensitivity analysis is performed. The impact of system characteristics and altering load on the power system's dynamic responsiveness is studied. The effect of system parameter and changing each load by $\pm 20 \%$ of their nominal value on the system response is investigated. Figures 4 and 5 show the gain of controller (optimised by MOGA and
MOPSO) and MPC computed in response to a $20 \%$ step load increase. It is demonstrated that the influence of system parameters and altering load on the system's dynamic performance is essentially non-existent. As a result, the suggested technique is stable under various situations, and the controller settings acquired under nominal circumstances do not need to be adjusted when a wide range of load and system parameter variations are applied. Two situations (figure 4 show Case 1 and figure 5 and Case 2) relating to RES fluctuation and severe parametric fluctuations (robustness analysis) are used to determine the superiority of the proposed model-based controller. The findings of Cases 1 and 2 show that, despite the high system complexity and fluctuation nature of the RESs, all of the developed MOGA and MOPSO controllers can successfully stabilize the grid frequency. When it comes to settling time and overshoot, the MOPSO and MOGA surpass the MPC and traditional PID. The peak overshoot and undershoot of frequency deviation for the two cases are compared using different controllers, as shown in Figures 4 and 5. In addition, Figures 4 and 5 show the percentage improvement of the recommended model-based controller over the MPC and traditional PID. The results show that the MOPSO controller outperforms the other two controllers. When the parametric variation is applied to the test MG, the performance of the developed MOPSO and MOGA controllers deteriorates, as evidenced by comparing the results of Case 1 and Case 2. The proposed technique, on the other hand, achieves a better level of stability than the other compared controllers.

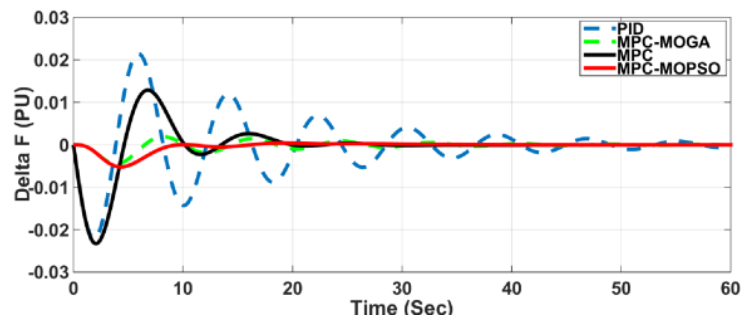

(a)

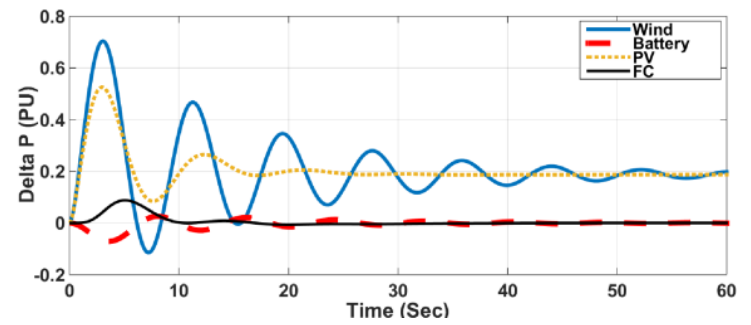

(b)

Fig. 3. In case of presence of the Wind/PV/FC/Battery energy sources, Frequency response in response to $2 \%$ step load increase (fixed parameters controllers and adaptive controller-based MOPSO and MOGA).

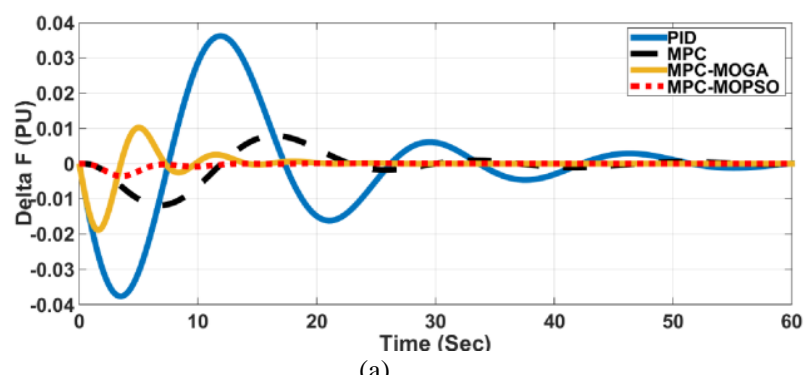

(a) 


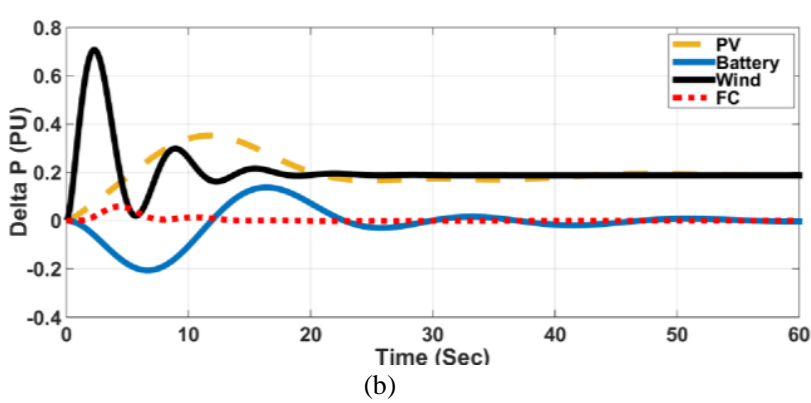

Fig. 4. In case of presence of the Wind/PV/FC/Battery energy sources, frequency response in response to $2 \%$ step load increase and wind turbine disturbance (fixed parameters controllers and adaptive controller-based MOPSO and MOGA).

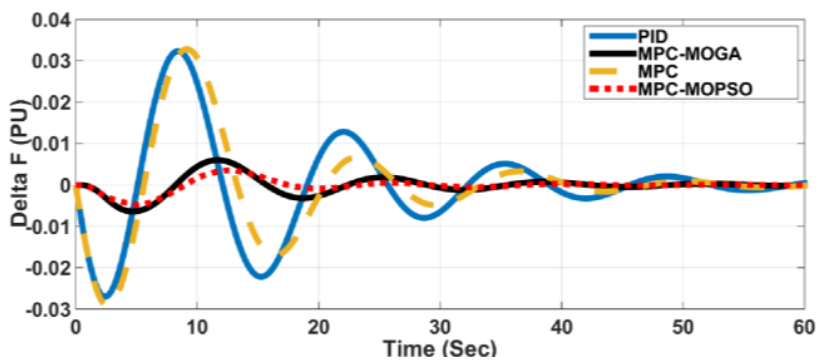

(a)

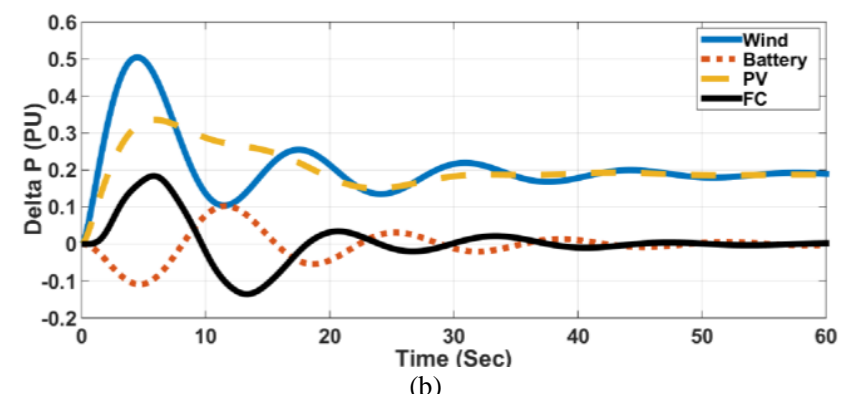

Fig. 5. Frequency response in case of presence of the Wind/PV/FC/Battery energy sources in response to $2 \%$ step load increase and PV disturbance (fixed parameters controllers and adaptive controllerbased MOPSO and MOGA).

\section{CONCLUSION}

The paper presents an adaptive load frequency control scheme using MOPSO based on centralized MPC control scheme in the power grid within largescale hybrid PV/Wind/FC generation and storage battery. The suggested controller was tested by incorporating a demand response programme in systems with high $\mathrm{PV} /$ wind turbine/FC penetration. The study proposes that MPC be used to regulate the output generation of each model, including hybrid renewable $\mathrm{PV} / \mathrm{Wind} / \mathrm{FC}$ with storage batteries, by estimating the future generation output value based on prior data and information. The gain of the system controller was tuned using the MOPSO based on MPC optimization technique. The wind speed was assumed to be changeable in the simulations performed in this work since wind turbines generate power with uncertainty and volatility in real-world situations. As a result, incorporating such a challenge into the suggested frequency control management control scheme was beneficial. The system with MOPSO was compared to the system with fixed controller settings in the case of step load change. The system with MOPSO may offer the best performance at the time of step load demand, according to digital simulation results. In addition, in the situation of frequency fluctuations caused by the hybrid PV/Wind/FC/Battery energy source, the system with MOPSO algorithm was contrasted to system with conventional controller. The MOPSO system, which is based on a centralised MPC control strategy, can improve system stability, robustness, and decrease LFC uncertainties induced by parametric and nonlinearity uncertainties in power systems. The system response increased significantly as a result of the wind farm's contribution to frequency management, since not only interchanging power and overshoot were reduced, but also the minimum or undershoot frequency was greatly enhanced. In addition, the system's settling time was shortened, and the system reached a more consistent and smooth steady state.

\section{REFERENCES}

[1] Chao Yang; Wei Yao; Ying Wang; Xiaomeng Ai "Resilient EventTriggered Load Frequency Control for Multi-Area Power System with Wind Power Integrated Considering Packet Losses" IEEE Access, 2021, Volume: 9.

[2] Yiwei Wu; J. Lim; Jian Shi "Stability-Constrained Microgrid Operation Scheduling Incorporating Frequency Control Reserve" IEEE Transactions on Smart Grid, 2020, Volume: 11, Issue: 2.

[3] Tohid Rahimi; Lei Ding; Mostafa Kheshti;Rasoul Faraji; Josep M. Guerrero;Gibran David Agundis Tinajero "Inertia Response Coordination Strategy of Wind Generators and Hybrid Energy Storage and Operation Cost-Based Multi-Objective Optimizing of Frequency Control Parameters" IEEE Access, 2021, Volume: 9.

[4] Ali Moeini; Innocent Kamwa "Analytical Concepts for Reactive Power Based Primary Frequency Control in Power Systems" IEEE Transactions on Power Systems, 2016, Volume: 31, Issue: 6.

[5] Ahmed Fathy; Abdullah G. Alharbi "Recent Approach Based Movable Damped Wave Algorithm for Designing Fractional-Order PID Load Frequency Control Installed in Multi-Interconnected Plants with Renewable Energy" IEEE Access, 2021, Volume: 9.

[6] Ahmad Karimi-Rizvandi; Mehrdad B. Sandjareh; Mohammad Hassan Nazari; Mohsen Ezati-Yaraziz "A Novel Frequency Control Scheme for Autonomous Microgrid Using Cooperative Application of Supercapacitor-Battery HESS, Photovoltaics, LED Lighting Loads and TCLs" IEEE Access, 2021, Volume: 9.

[7] Ioannis D. Margaris; Stavros A. Papathanassiou; Nikos D. Hatziargyriou; Anca D. Hansen; Poul Sorensen "Frequency Control in Autonomous Power Systems with High Wind Power Penetration" IEEE Transactions on Sustainable Energy, 2012, Volume: 3, Issue: 2.

[8] Masoud H. Nazari; Le Yi Wang; Santiago Grijalva; Magnus Egerstedt "Communication-Failure-Resilient Distributed Frequency Control in Smart Grids: Part I: Architecture and Distributed Algorithms" IEEE Transactions on Power Systems, 2020, Volume: 35, Issue: 2.

[9] Zhiying Wu; Huadong Mo; Junlin Xiong; Min Xie "Adaptive EventTriggered Observer-Based Output Feedback L $\infty$ Load Frequency Control for Networked Power Systems" IEEE Transactions on Industrial Informatics, 2020, Volume: 16, Issue: 6 .

[10] Chaoxu Mu; Yong Zhang;Hongjie Jia;Haibo He "Energy-StorageBased Intelligent Frequency Control of Microgrid With Stochastic Model Uncertainties" IEEE Transactions on Smart Grid, 2020, Volume: 11, Issue: 2.

[11] Arman Sargolzaei; Kang K. Yen; Mohamed N. Abdelghani "Preventing Time-Delay Switch Attack on Load Frequency Control in Distributed Power Systems" IEEE Transactions on Smart Grid, 2016, Volume: 7 , Issue: 2 .

[12] Fernando D. Bianchi; Jos Luis Domnguez-Garca "Coordinated Frequency Control Using MT-HVDC Grids With Wind Power Plants" IEEE Transactions on Sustainable Energy, 2016, Volume: 7, Issue: 1

[13] H. Bevrani; F. Habibi; P. Babahajyani; M. Watanabe; Y. Mitani "Intelligent Frequency Control in an AC Microgrid: Online PSO-Based Fuzzy Tuning Approach" IEEE Transactions on Smart Grid, 2012, Volume: 3, Issue: 4.

[14] Luis M. Castro; Claudio R. Fuerte-Esquivel; J. Horacio TovarHernandez "Solution of Power Flow with Automatic Load-Frequency Control Devices Including Wind Farms" IEEE Transactions on Power Systems, 2012, Volume: 27, Issue: 4.

[15] Sonalika Mishra; Pratap Chandra Nayak; Umesh Chandra Prusty; Ramesh Chandra Prusty "Model predictive controller based load 
frequency control of isolated microgrid system integrated to pluggedin electric vehicle" 2021 1st Odisha International Conference on Electrical Power Engineering, Communication and Computing Technology (ODICON), 2021.

[16] Subhranshu Sekhar Puhan; Renu Sharma; Saumya Ranjan Lenka "Frequency control through BESS and SOFC based fuel cell in a Mult source Microgrid" 2021 1st Odisha International Conference on Electrical Power Engineering, Communication and Computing Technology(ODICON), 2021.

[17] Mohammad Saeid Akbari; Ali Akbar Safavi; Navid Vafamand, Tomislav Dragičević; Jose Rodriguez "Fuzzy Mamdani-based Model Predictive Load Frequency Control" 2020 IEEE 11th International Symposium on Power Electronics for Distributed Generation Systems (PEDG), 2020.

[18] Shoults R R, Jativa Ibarra J A. Multi area adaptive LFC developed for a Comprehensive AGC simulation. IEEE Trans Power System 1993; 8(2): 541-547.

[19] Chaturvedi D K, Satsangi P S, Kalra P K. Load frequency control: a generalized neural network approach. Int. J. Elect. Power Energy System 1999; 21(6): 405-415.
[20] Ghosal S P. Optimization of PID gains by particle swarm optimization in fuzzy based automatic generation control. Electrical Power System Research 2004; 72: 203-212.

[21] Khuntia S R, Panda S. Simulation study for automatic generation control of a multi-area power system by ANFIS approach. Applied Soft Computing 2012; 12(1): 333-341.

[22] Nanda J, Mishra S, Saikia L C. Maiden application of bacterial foraging based optimization technique in multiarea automatic generation control. IEEE Trans Power System 2009; 24(2): 602-609.

[23] Rout U K, Sahu R K, Panda S. Design and analysis of differential evolution algorithm based automatic generation control for interconnected power system. Ain Shams Engineering Journal 2013; 4(3): 409-421.

[24] Singh Parmar K P, Majhi S, Kothari D P. Load frequency control of a realistic power system with multisource power generation. Int. J. Elect. Power Energy System 2012; 42(1): 426-433. 